\title{
Beam Combination for Stellar Imager and its Application to Full-Aperture Imaging
}

\author{
D. Mozurkewich ${ }^{a}$, K. G. Carpenter ${ }^{b}$, R. G. Lyon ${ }^{b}$ \\ ${ }^{a}$ Seabrook Engineering, 9310 Dubarry Ave, Seabrook MD, 20706 USA \\ ${ }^{b}$ NASA/Goddard Space Flight Center, Greenbelt MD, 20771 USA
}

\begin{abstract}
Stellar Imager (SI) will be a Space-Based telescope consisting of 20 to 30 separated apertures. It is designed for UV/Optical imaging of stellar surfaces and asteroseismology. This report describes details of an alternative optical design for the beam combiner, dubbed the Spatial Frequency Remapper (SFR). It sacrifices the large field of view of the Fizeau combiner. In return, spectral resolution is obtained with a diffraction grating rather than an array of energy-resolving detectors. The SFR design works in principle and has been implemented with MIRC at CHARA for a small number of apertures. Here, we show the number of optical surfaces can be reduced and the concept scales gracefully to the large number of apertures needed for Stellar Imager.

We also describe a potential application of this spatial frequency remapping to improved imaging with filledaperture systems. For filled-aperture imaging, the SFR becomes the core of an improved aperture masking system. To date, aperture-masking has produced the best images with ground-based telescopes but at the expense of low sensitivity due to short exposures and discarding most of the light collected by the telescope. This design eliminates the light-loss problem previously claimed to be inherent in all aperture-masking designs. We also argue that at least in principle, the short-integration time limit can also be overcome. With these improvements, it becomes an ideal camera for TPF-C; since it can form speckle-free images in the presence of wavefront errors, it should significantly relax the stability requirements of the current designs.
\end{abstract}

Keywords: imaging interferometry, UV/Optical, space-based, coronagraph

\section{INTRODUCTION}

Stellar Imager is a proposed UV/optical, space-based interferometer designed for spectral imaging of stellar surfaces and asteroseismology (Carpenter VT XL, these proceedings). It is composed of 20 to 30,1 meter free-flying apertures and a beam combiner in a central hub. In this paper, we suggest replacing the Fizeau beam combiner advocated for the Stellar Imager Mission (http://hires.gsfc.gov/si/) with a design dubbed the Spatial Frequency Remapper, SFR, which maps the spatial frequencies measured by the apertures into a onedimensional configuration, then uses the second dimension of a two-dimensional detector for spectral information. The concept is presented in Section 2, a design consistent with stellar imager is presented in Section 3. That design is robust, can accommodate a large number of apertures, accept either focal or afocal beams and can be implemented with a small number of optical surfaces. The emphasis changes to ground-based applications in Section 4 where the SFR is used as the core of a high-precision imaging system for full-aperture telescopes.

\section{BASIC CONCEPTS}

A schematic representation of the concept is shown in shown Figure 1. The beams from the apertures are arranged in a non-redundant, one-dimensional pattern. The final lens brings them to a common image which consists of an Airy disk of a single aperture, crossed with a fringe pattern from each pair of apertures. Since no two apertures have the same spacing, no two fringe frequencies are the same. The amplitude and phase of the fringes can be determined in post processing. This arrangement needs a one-dimensional array detector with pixels as large as the Airy disk in one dimension but fine enough to resolve the highest-frequency fringe in the

Send correspondence to David Mozurkewich

E-mail: dave@mozurkewich.com 

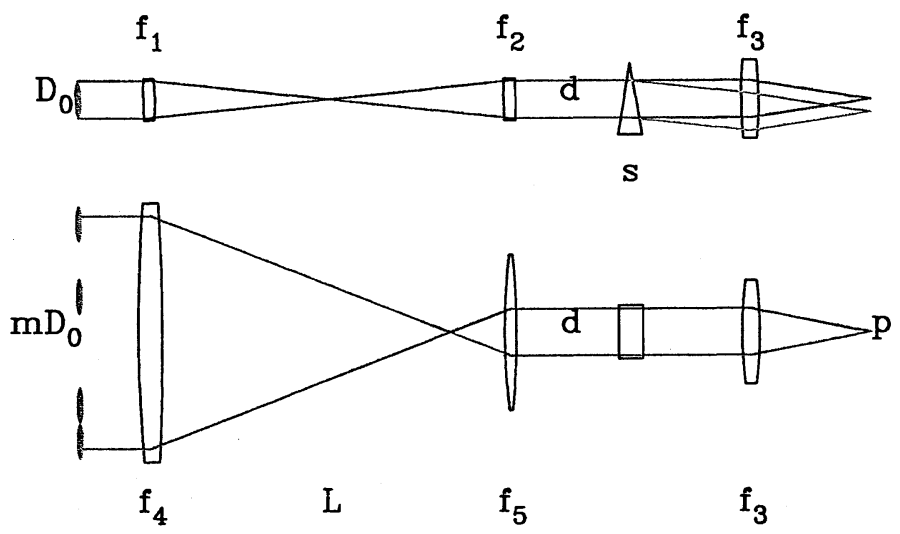

Figure 1. Conceptual design for the spatial frequency remapper optics. Two orthogonal views of the system are shown. The first two elements are cylindrical and form a beam compressor with high compression in the fringe direction (lower plot) and low compression in the cross fringe direction (upper plot). The dispersing element, shown as a prism, disperses the light in the cross fringe direction. The final lens images the light onto the detector, which sees fringes in one direction and a spectrum in the orthogonal direction.

orthogonal direction. Since pixels are usually square, the lens is preceded by an anamorphic beam compressor which compresses the beam by different amounts in the fringe and spectral directions. Finally, a prism is added to disperse the light perpendicular to the fringes. The result is a frame of data where each column can be Fourier transformed to determined the fringe parameters at a different wavelength.

The most challenging part of the SFR is the anamorphic beam compressor. Anamorphic systems are difficult to build for a typical application requiring a large field of view. This system, however, is strictly on-axis since the sources being observed are smaller than the diameter of the Airy disk. A pair of biconic* (astigmatic) parabolas can be used to perform a perfect (no aberrations) transformation between afocal beams. The anamorphic magnification required for the 30-aperture system needed for Stellar Imager is 1360. Although this design can be manufactured, we choose to divide the apertures into two groups of 15 each and allow the combiner to form separate data frames for the two groups of apertures. This reduces the number of detector pixels needed by a factor of two and the anamorphic magnification by a factor of four (to 303). It also reduces the number of measured baselines by a factor of two but since this two-combiner design also increases the signal to noise per baseline $^{\dagger}$, the missing measurements can be regained by changing which apertures feed which combiner group. Even though the baselines are not all measured at once, there is, at least in principle, no loss of throughput by doing so.

It is not possible to measure all baselines with one reconfiguration; three configurations are needed. To see this, divide the apertures into four subgroups labeled A, B, C, D. The three required configurations are formed by consecutively combining subgroups $\mathrm{A}$ and $\mathrm{B}, \mathrm{A}$ and $\mathrm{C}$ and $\mathrm{A}$ and $\mathrm{D}$ into one group while the remaining apertures form the other group. Baselines formed from two apertures within a subgroup are measured in all three configurations while the remaining baselines are measured once. The resulting factor of $\sqrt{3}=1.7$ signal to noise variation between baselines can be put to good use. Depending on the needs of the observer, either the important, low signal to noise baselines can be observed in each configuration to improve those data or the short, higher amplitude baselines can be measured in all three configurations and used by the control system to keep the array in phase. An additional advantage of the two-group design is fault tolerance. If one combiner should

${ }^{*}$ The shape of these two mirrors is given by $z=x^{2} / 2 f_{x}+y^{2} / 2 f_{y}$. They are the parabolas needed to provide the desired compression separately along the $x$ and $y$ axes.

${ }^{\dagger}$ Removing half the apertures from a combiner removes half of the photon noise variance but does not change the amplitude of the fringes that are still present. Fewer baselines are measured but those present have higher signal to noise per unit time. 


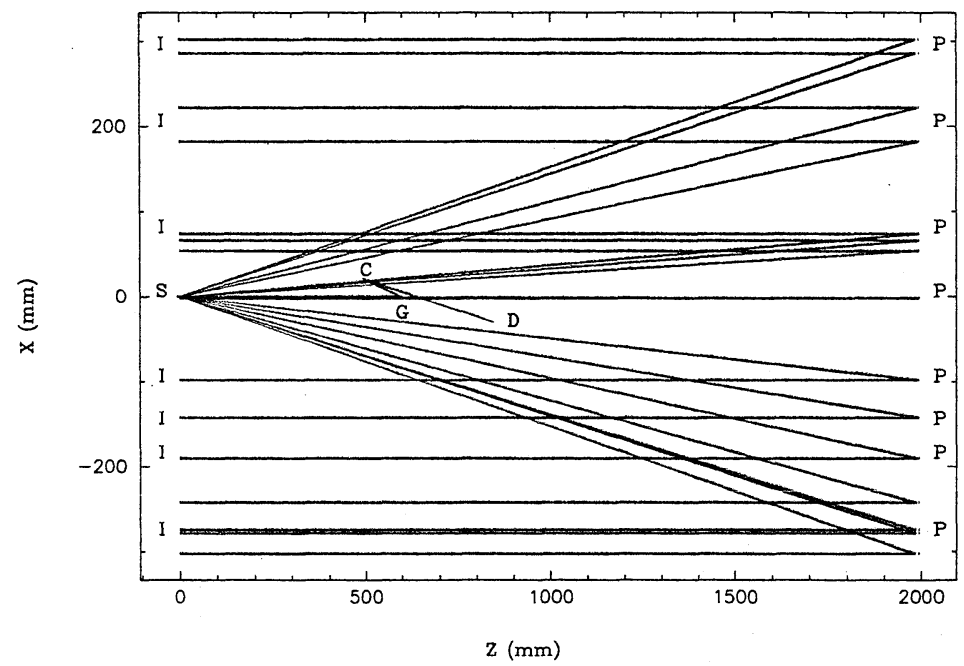

Figure 2. SFR beam compressor design for Stellar Imager, plan view. The 30 input beams are divided into two rows. The 15 beams in each row are spread out from top to bottom of this figure. The primary, $P$, shown as individual segments for each aperture, compresses the beams and directs them to the secondary, $S$, where they are recollimated. The grooves of the diffraction grating, $G$, run parallel to the $X$ axis and diffract the light into the page. That tilt of the beam is not seen in this figures, but the grating is also tilted about the $Y$ axis to deflect the light up to the camera parabola, $C$, and finally the detector $D$. Note that the $X$ and $Z$ scales are different.

fail, the mission continues but with half the throughput of the full mission.

\section{DESIGN FOR STELLAR IMAGER}

An SFR design that is a good fit to the Stellar Imager mission is shown in Figures 2 and 3 in orthogonal views. The specifications for the optics are given in Table 1. The afocal input beams, labeled with $I$ and $2 \mathrm{~mm}$ in diameter, enter from the left starting at $Z=0$. They are arranged in two rows of 15 and propagate to the right in both figures to $Z=2000$. Mirrors at $Z=2000$ and $Z=0$ are the primary, $P$, and secondary, $S$, of an anamorphic beam compressor; the beam diameters and their spacings are compressed in the $X$ direction while they are left unchanged in the $Y$ direction. Although the primary is shown as 30 individual mirror segments, all are part of a single, biconic parabola. The collimated output of this beam compressor is parallel to the input beams. Two diffraction gratings, $G$, are located at $Z=587$, one for each row of 15 beams. They are aligned with their grooves parallel to the $\mathrm{X}$-axis. The camera consists of two parabolas, $C$, one for each row of 15 beams, and a detector $D$. The detector sees two separated fringe patterns. The patterns are separated by tilting the gratings by different amounts and translating the parabolas perpendicular to their optical axes. Image quality is good: the rms image size is less then $10 \mu \mathrm{m}$ with $15 \mu \mathrm{m}$ pixels Nyquest sampling the image.

In addition to the beam combiner, the entire optical train contains two or three additional mirrors for each aperture: the primary mirror segment, and conditioning mirrors in the hub. The conditioning mirrors provide control of tip, tilt and piston, as well as adjusting the lateral pupil position inside the combiner. For this design, they also collimate the beam. If the collimation is not possible with the conditioning mirrors, the shape of the primary mirror segments of the SFR can be modified to take on that task. It is also possible to reduce this design to three surfaces by shaping the secondary of the beam compressor to generate a converging beam. Then a holographic grating in that converging beam gives the spectral resolution. This three-surface design was generated by treating the fringe and spectral directions as independent, symmetric systems to determine the focal lengths and conic constants. Then, they were combined to generate the anamorphic system. The spot diagram for this three surface system is show in Figure 4. The performance is good; the rms spot size is less than $5 \mu \mathrm{m}$ with $15 \mu \mathrm{m}$ pixels Nyquest sampling the beam. 


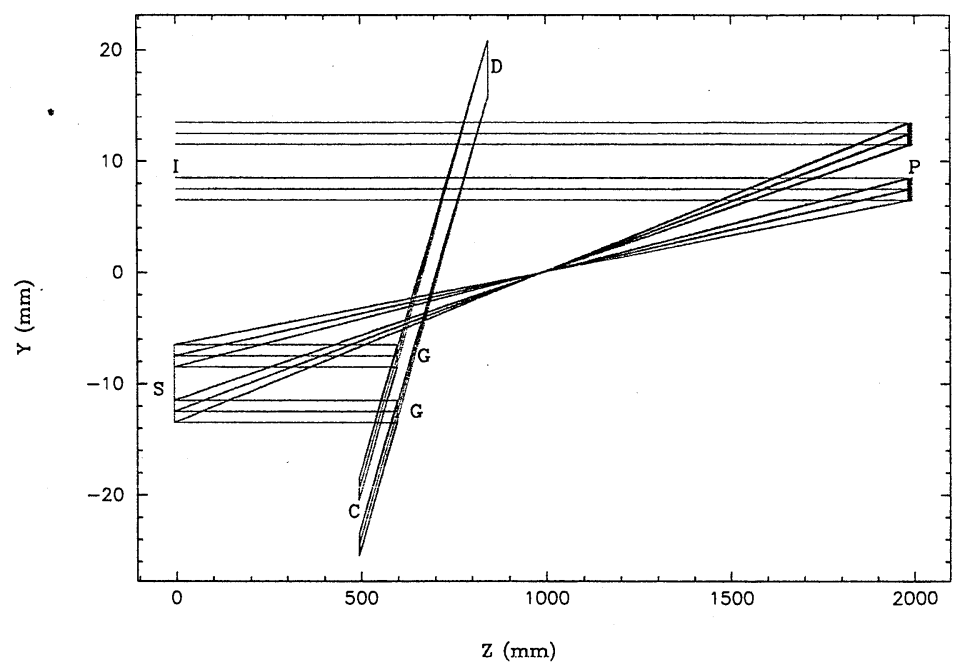

Figure 3. SFR beam compressor design for Stellar Imager, side view. The beams enter from the left at $I$ and travel to the right. Two rows of 15 beams each are seen edge on. The primary, $P$, of the anamorphic beam compressor is shown in the upper right. The secondary, $S$, in the lower left recollimates the beams. Diffraction gratings, $G$, with their grooves parallel to the $X$ axis, disperse the light to the lower left where parabolas, $C$, form an image at the detector $D$. Two fringe patterns are formed; one for each row of beams. Note that the $Y$ scale differs from the $X$ scale in Figure 2.

Table 1. Beam Combiner Parameters

\begin{tabular}{|l|rrr|}
\hline & f.l. & Dia. & off axis \\
\hline Primary & 1993.421 & 606.0 & 0.0 \\
& 1000.000 & 7.0 & 10.0 \\
\hline Secondary & 6.579 & 2.0 & 0.0 \\
& 1000.000 & 7.0 & 10.0 \\
\hline Imaging parabola & 355.556 & 2.0 & 20.0 \\
& 355.556 & 2.0 & 0.0 \\
\hline
\end{tabular}

The need for 3 configurations per image should not have a significant impact on mission overhead; the position of the primary mirror segments need not change between configurations; half of the mirrorsats have to tilt in order to feed the other combiner. This tilt is about a milliradian and is usually less; it should not consume significant time compared to the total integration time and the time to change pointing to another star. An active light baffle will be needed since each of the configurations will require holes in different places. This reconfiguring of the baffle is not in the baseline design but should be a simple addition and is probably a good idea even with a Fizeau combiner.

\section{APPLICATION OF INTERFEROMETRY TO FULL-APERTURE IMAGING}

It is possible to apply interferometric imaging techniques to a full-aperture telescope. The idea is to divide the telescope aperture into a number of interferometric apertures, detect fringes between the interferometric apertures and form images from those data. Called aperture masking, this technique has been successfully used. In the past, the interferometric apertures were formed using a two-dimensional, non-redundant mask placed over one of the telescope's mirrors. Though the technique has low sensitivity, the resulting images are high quality, typically better than those obtained with adaptive optics. Low sensitivity results from the short integration times needed to freeze atmospheric turbulence and the low photon rate caused by the mask blocking most of the 


\section{Spot Diagram: Surface 4}

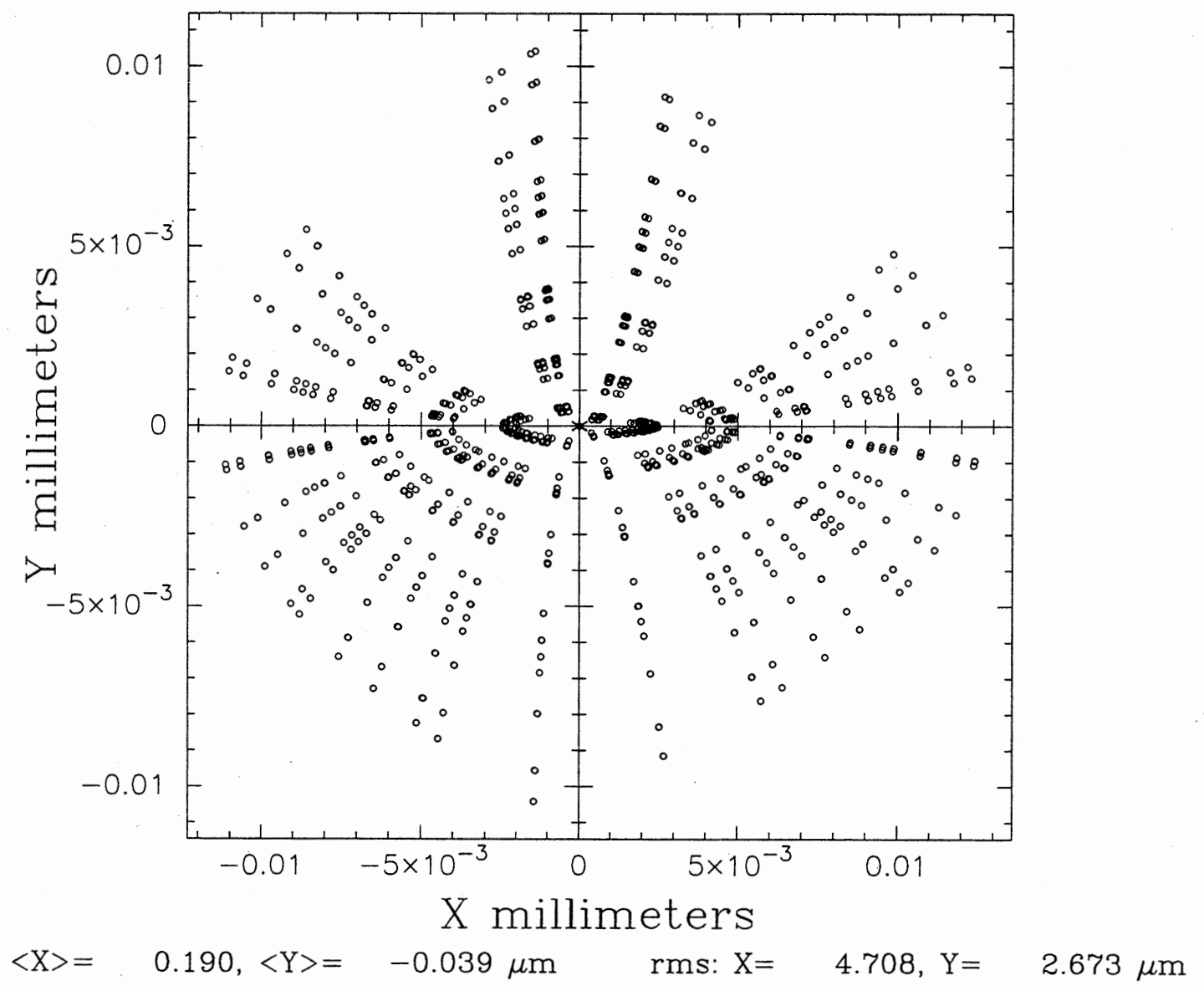

Figure 4. A spot diagram at the detector for the central wavelength. The pixels, which Nyquest sample the image are $15 \mu \mathrm{m}$ across. The azimuthal gaps may seem mysterious but they correspond to the gaps in the one-dimensional configuration. The rays from each aperture form a U-shaped pattern. The U straddles the Y-axis with its base at the origin. 
aperture and the use of a narrow bandpass to prevent problems due to phase variation with wavelength. The improved performance comes from the use of closure relationships which generate nearly perfectly calibrated visibility phases. Better visibility amplitudes result from the use of a non-redundant aperture. The amplitude calibration is not perfect but it is better than the calibration of the amplitudes from an adaptive optics (AO) system. With $\mathrm{AO}$, the wavefront errors are reduced, but not eliminated. Multiple instances of the same baseline within the full aperture therefore have different phases and since they are combined by the telescope before detection, the fringe amplitude is reduced.

Most, if not all of these shortcomings can be eliminated while preserving the interferometric quality of the image. The full aperture is divided into interferometric apertures using a lenslet array. The lenslets feed single-mode fibers which rearrange the apertures into a one-dimensional, non-redundant configuration. That configuration is then fed through a SFR-style beam combiner.

The image quality should be improved over a normal aperture masking system. In both cases, closure relationships provide well-calibrated phases. The new system has the additional advantage of spatial filtering thanks to the single mode fibers. Wavefront errors across an interferometric aperture are converted into intensity errors. The intensity errors can be measured or alternatively, additional closure relationships can be applied to form high-precision amplitudes. These closure amplitudes are analogous to closure phases; as long as the visibility amplitude on a baseline between stations $i$ and $j$ can be written as $V_{i, j}=g_{i} g_{j} \Gamma_{i, j}$, where $V$ is the observed visibility amplitude, $\Gamma$ is the correct value and the gains, $g$, depend only on the station, then the $N(N-1) / 2$ amplitudes can be calibrated by specifying $N$ gains leaving $N(N-1) / 2-N$ good amplitudes. Intensity errors manifest as non-unit gains.

A less-obvious but more important improvement occurs if this full-aperture imaging system is placed behind an adaptive optics system - much of the temporal variation of the visibility phase is due to the low-order aberrations across the aperture which the AO system is good at removing. With AO, longer integration times should be possible. How long can the integration time be? This is still an open question. Since spatial filtering does not correct for temporal phase variations within an integration, it is usually assumed the integration time must be short enough to freeze the atmosphere. But that is not necessarily the case. If the AO system has a high Strehl, the integration times may be very long indeed because the combination of spatial filtering and AO should allow the closure amplitudes to correct for temporal phase variations during the integration. By removing the low-order aberrations across the telescope, adaptive optics helps decorrelate temporal variations between the interferometric apertures. To the extent that the temporal phase variations are Gaussian and independent, the visibility amplitude reduction can be written in terms of gains, $g_{i}=e^{-\sigma_{i}^{2}}$, where $\sigma_{i}^{2}$ is the phase variance for aperture $i$. As long as the AO Strehl is sufficiently high (a low Strehl implies a large $g_{i}$ which hurts sensitivity), very long integration times may be possible resulting in good sensitivity and very high precision simultaneously.

A possible criticism of this device is the need to deal with a very large number of apertures. The diameter of the field of view, measured in resolution elements, is the ratio of the highest to lowest spatial frequency. The highest is given by the diameter of the telescope; the lowest by the diameter of the beams collected by the lenslets. Frequencies lower than the diameter of the lenslet are lost. Thus a large field of view requires a large number of relatively small apertures. The lenslets should be in a hexagonal pattern (close packed). A lenslet array with a center element and 5 rings has a field of view of eleven times the resolution and 91 apertures. Discarding the central aperture since it will almost always be blocked by the secondary ${ }^{\ddagger}$, there are 90 usable apertures and 4005 baselines. The shortest known one-dimensional, non-redundant configuration for 90 apertures is of length 6967. Taking 4 pixels per fringe, an array detector with a long dimension of 27,868 pixels is required. A detector this size is challenging, but perhaps not out of the question. At least as challenging is the fiber array with the shortest output spacing being twice the diameter of the output beams, the ratio of longest spacing to beam diameter is 13,934-100 micron diameter beams would require a 1.4 meter diameter mirror for the beam combiner. We risk having the SFR optics approach the size of the telescope.

The solution starts with the realization that the final signal to noise depends very weakly on how we combine the light from the $N$ collectors to form fringes. One combination scheme is to combine all $N$ beams on a single detector. This allows us to measure the amplitude and phase of $N(N-1) / 2$ fringes simultaneously. If instead,

\footnotetext{
${ }^{\ddagger}$ A larger central obscuration is not a problem; there will simply be fewer baselines with data.
} 


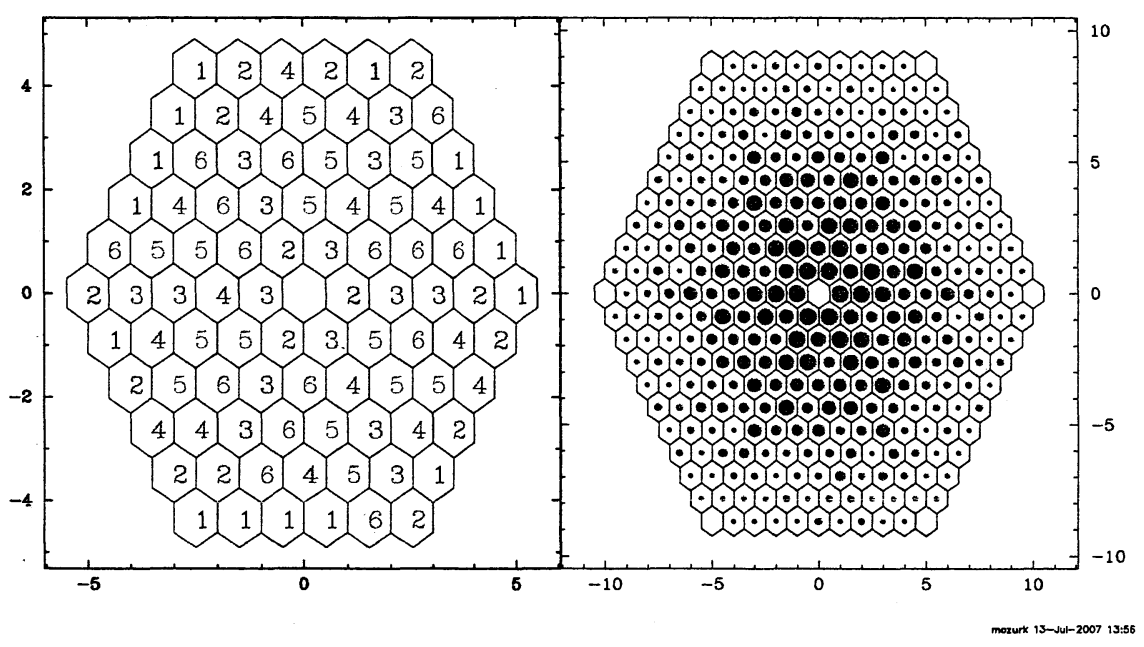

Figure 5. One possible configuration for the full-aperture interferometric imaging system. The telescope pupil is shown on the left, divided into 90 apertures with a lenslet array. The color and number in the cell indicates the combiner to which light from that aperture is delivered. The MTF is shown on the right with the size of the dot showing the redundancy. The maximum redundancy is 12 .

we build two beam combiners that each take $N / 2$ beams, the number of fringes per combiner is reduced by $2(N-1) /(N / 2-1)$, approximately a factor of four. Since we have two combiners, fringes on half of the baselines are observed. The signal is the number of photons that contribute to a particular fringe pattern and does not depend on how many beams are combined. The noise, however, is the square root of the total number of photons and is reduced by a factor of $\sqrt{(2)}$ when two combiners are used instead of one. With $\sqrt{2}$ less noise, the same signal to noise can be obtained with half the observing time and the observing time per baseline measured is the same for both designs.

The second important realization stems from the fact that a close-packed, hexagonal array is highly redundant; since there are many more measurements than independent spatial frequencies, fringe measurements between all pairs of apertures are not needed; the goal is simply to use all the beams to measure all spatial frequencies about the same number of times. One solution for the 5 ring, hexagonal geometry considered above is to divide the apertures into 6 groups of 15. Fifteen-aperture groups were chosen to match the SFR design presented in the previous section. Six beam combiners are needed but with multiple groups using the same optics, the whole system can be relatively compact. With about 150 spectral channels, all six data frames should fit on a single $1 \mathrm{k}$ by $1 \mathrm{k}$ detector. Compared to the original design, this multi-frame design is much more compact. Using 0.5 $\mathrm{mm}$ diameter lenslets, the output side of the coherent fiber bundle is only $22 \mathrm{~cm}$.

Although all goals cannot be realized; this one can. One multiple-combiner configuration consistent with our requirements is shown in Figure 5. The left-hand plot shows the full aperture telescope divided into 91 apertures with lenslets. Light hitting the central lenslet is disposed of while the other lenslets are divided into 6 groups of 15 each. The numbers identify which group that lenslet feeds. The right-hand side shows the resulting MTF; the size of the spot in each cell increases with the redundancy of that measurement. Only the corner cells are missing; these need not be included within the area of the telescope aperture. The redundancy increases from one for the longest baselines to 12 for some of the shortest baselines. If all the apertures were delivered to a single combiner, the shortest baselines would be measured 78 times while the longest baselines would be measured 1 to 6 times depending on how far the baseline is from the corners of the outer ring. Thus compared to a standard, filled aperture image, this 6-combiner configuration gives more weight to the high spatial frequencies, a feature that is probably desirable. 


\section{APPLICATION OF SFR TO CORONAGRAPHY}

The device just described will have an obvious NASA application when a program like the Terrestrial Planet Finder - Coronagraph is reinstated. The performance of the TPF-C designs is not limited by the amount of starlight rejection the coronagraph provides; rather it is the stability of the residual stellar wavefront. The residual starlight forms speckles in the image, any of which can be mistaken for a planet. The proposed observational mode for TPF-C is to take several images, rotating the spacecraft between images. The planet can be separated from the speckles because the position of the planet is fixed relative to the sky while the speckles are fixed relative to the spacecraft. For this technique to work, the wavefront has to be stable over times scales of an hour while the spacecraft is rotated for the images. The cost of the mission is driven as much by the need for stability as it is by the amount of starlight rejection that can be achieved.

Instead of relying on stability, one can place the aperture-masking SFR described in the previous paragraphs after the coronagraph to work as both the imaging sensor and a wavefront sensor. Since this detector uses the entire aperture, there is no significant SNR loss from using it. With most of the starlight already rejected by the coronagraph, the dynamic range needed to detect a planet is in the range of 1000 , easily achieved by aperture masking.

As long as the phase errors are small, changes in phase during the integration time are averaged to produce a mean phase. Since the process is linear and the integration time is the same for all spatial frequencies this has no effect on the closure phases. Phase and amplitude errors in the wavefront affect the phase and amplitude of the spatial frequency quadratically but since we are starting from a very good wavefront, these effects should be small.

\section{ACKNOWLEDGMENTS}

The research was supported by NASA contract NNX07CA49P. 